\title{
Consecuencias de la restricción de peso impuesta por el deporte en la infancia
}

\author{
Nathalie Boisseau \\ LAPHAP, Faculté des Sciences du Sport, Poitiers, Francia
}

\section{Palabras clave}

Deporte en la infancia - Restricción de peso · Crecimiento, infancia $\cdot$ Maduración

\section{Extracto}

Actualmente, los competidores jóvenes emprenden programas de entrenamiento que se basan en un nivel elevado de actividad física desde una temprana edad. Este entrenamiento intensivo (volumen e intensidad) aumenta el gasto de energía, que tiene que ser compensado por una mayor ingesta nutricional. Los bailarines de ballet, los patinadores artísticos, los gimnastas o los gimnastas rítmicos comienzan a entrenar a los 5 o 6 años de edad, y la mayoría practica durante más de 20 a 30 horas por semana. En estas actividades, así como en los deportes clasificados por peso (luchadores, jinetes y atletas practicantes de judo o boxeo), los jóvenes atletas de elite adoptan decisiones conscientes para reducir su ingesta alimentaria con objeto de mantener la esbeltez, el físico prepuberal o para 'dar el peso'. El consumo adecuado de energía y micronutrientes es esencial para los niños en fase de crecimiento, y las restricciones calóricas y de líquidos durante el entrenamiento físico en la infancia o durante la adolescencia podrían perturbar las regulaciones metabólicas y hormonales que influyen sobre el crecimiento, la maduración, la composición corporal, el ciclo menstrual y la capacidad reproductora, lo que puede incrementar el riesgo de traumatismos como fracturas por sobrecarga. Además, estas estrategias pueden generar trastornos del comer (anorexia y/o bulimia nerviosa), especialmente durante la adolescencia femenina, afectando a la imagen corporal, a la imagen de uno mismo y ocasionando inadaptaciones sociales y emocionales.

Copyright @ 2006 Nestec Ltd., Vevey/S. Karger AG, Basel

\section{KARGER \\ Fax +41613061234 \\ E-Mail karger@karger.ch}

www.karger.com (c) 2006 Nestec Ltd., Vevey/S. Karger AG, Basel

0252-8185/06/0642-0077\$23.50/0

Accesible online en:

www.karger.com/ans

\section{Introducción}

Durante la infancia y la adolescencia, el crecimiento y el desarrollo rápidos incrementan las necesidades de energía y micronutrientes. Las necesidades nutricionales son mayores en atletas jóvenes debido al nivel superior de actividad física. Las restricciones de alimentos y líquidos en atletas de elite jóvenes son frecuentes, especialmente en deportes estéticos y de clasificación por pesos, y pueden ser potencialmente peligrosas durante el período de crecimiento. El objetivo de esta revisión es informar sobre los efectos adversos potenciales para la salud de la restricción de peso impuesta por el deporte en la infancia.

\section{Restricciones de alimentos y líquidos en categorías deportivas específicas}

\section{Energía, macronutrientes y micronutrientes}

Durante la infancia y la adolescencia, el crecimiento y el desarrollo rápidos influyen sobre las necesidades de energía, macronutrientes y micronutrientes.

\section{Energía}

Debido a la considerable variabilidad intraindividual e interindividual, nos encontramos con ciertas dificultades para cuantificar las necesidades energéticas estimadas en el niño o adolescente atlético o muy activo. El cálculo de la estimación de las necesidades energéticas por parte de la Junta de Alimentos y Nutrición se basa en ecuaciones que comprenden la edad, la talla, y el peso 
corporal de un individuo, así como una clasificación física de hábitos de vida sedentarios, moderadamente activos, activos o muy activos [1]. En niños de 9 a 13 años de edad, las necesidades energéticas fluctúan desde $1.415 \mathrm{kcal} /$ día en niñas sedentarias de 9 años hasta $3.038 \mathrm{kcal} /$ día en niños muy activos de 13 años. En adolescentes de 14 a 18 años, las necesidades energéticas fluctúan desde $1.718 \mathrm{kcal} /$ día en niñas sedentarias de 14 años hasta $3.804 \mathrm{kcal} /$ día en adolescentes muy activos de 18 años. Por lo tanto, el gasto total de energía en atletas infantiles y adolescentes está vinculado a la energía basal, el crecimiento y el nivel de actividad física. Por ejemplo, en gimnastas de elite, el entrenamiento físico incrementa las demandas calóricas e induce una ingesta adicional de 400 a 700 kcal de energía al día [2, 3].

\section{Consumo de energía en deportes con restricción de peso}

Con objeto de seguir delgados y mantener un físico juvenil, numerosos gimnastas de elite, gimnastas rítimicos, bailarines de ballet y patinadores artísticos están convencidos de que necesitan seguir una dieta. En los deportes basados en la clasificación por pesos (luchadores y atletas practicantes de judo o boxeo), las restricciones de la ingesta de alimentos y líquidos son practicadas corrientemente durante toda la temporada competitiva con objeto de competir en una a tres clases de peso por debajo de su peso normal [4], pensando que, de este modo, se obtendrá una ventaja competitiva sobre oponentes de menor tamaño. Por lo tanto, los ciclos repetidos de pérdida y recuperación de peso son habituales, y las ingestas de energía se perturban regularmente $[5,6]$. Estos 'recortes y recuperaciones del peso' se han vinculado a una reducción de las necesidades de energía debido al menor índice metabólico de los atletas jóvenes [7].

Aunque de forma limitada, en algunos estudios se ha demostrado que desde el principio del programa de entrenamiento (gimnastas, gimnastas rítmicos, bailarines de ballet, etc.) o durante toda la temporada de competición en el caso de atletas clasificados por peso, la ingesta de energía es teóricamente insuficiente para sustentar el crecimiento y un entrenamiento vigoroso [8-20].

\section{Proteína}

Es necesaria una ingesta suficiente de proteína para proporcionar aminoácidos esenciales que sustenten el crecimiento y, especialmente, el mantenimiento y el desarrollo de masa corporal magra. De hecho, el metabolismo proteínico es importante para el crecimiento y el remodelado del tejido magro. Un entrenamiento para ejercicios intensos y regulares incrementa las necesidades diarias de proteínas en atletas adultos $[1,21]$. Las ingestas de proteínas recomendadas en niños y adolescentes sedentarios o moderadamente activos (de edades entre $9 \mathrm{y}$ 18 años) se sitúan entre 0,73 y $0,85 \mathrm{~g} / \mathrm{kg}$ [1] o entre 0,80 y $1,0 \mathrm{~g} / \mathrm{kg}$ de masa corporal $[22,23]$. No obstante, esta recomendación no está basada en niños activos o muy activos. En realidad, no se dispone de datos sobre las necesidades proteínicas de atletas jóvenes. En la mayoría de los casos, los competidores jóvenes incrementan espontáneamente su ingesta de alimentos para obtener energía y satisfacen de un modo natural sus necesidades de proteínas. En deportes que conllevan comportamientos de restricción de alimentos, el metabolismo de las proteínas podría estar trastornado y, por tanto, deteriorar el crecimiento y/o la masa magra total.

En gimnastas o bailarinas, aunque las evaluaciones nutricionales indican que muchas de estas jóvenes reciben una ingesta calórica inferior a la ingesta diaria recomendada [1] para su edad y nivel de actividad física [17, 24-27], el consumo de proteínas parece exceder espontáneamente de 0,8 a $1,0 \mathrm{~g} / \mathrm{kg} /$ día, y en la mayoría de las ocasiones alcanza 1,3 a $1,8 \mathrm{~g} / \mathrm{kg} /$ día. De hecho, tal como se ha descrito en diferentes estudios nutricionales, estos atletas consumen un exceso de proteínas a expensas de la grasa y los hidratos de carbono [27]. Además, en un estudio reciente basado en el uso del isótopo estable ${ }^{15} \mathrm{~N}$-glicina se observa que el entrenamiento no afecta al recambio proteínico en gimnastas femeninas prepuberales y al inicio de la pubertad [17].

La actitud en relación con los luchadores o los atletas practicantes de judo de elite jóvenes es bastante diferente. En consecuencia, en diferentes publicaciones se ha demostrado que la situación de las proteínas nutricionales puede alterarse durante el período de 'recorte del peso' [28], que podría inducir un balance nitrogenado negativo transitorio conducente a posibles trastornos del crecimiento y lesiones musculares. Recientemente, Friedlander et al. [29] comunicaron que una restricción calórica durante 3 semanas altera el metabolismo de las proteínas (recambio y oxidación de la leucina) en hombres jóvenes con peso normal.

\section{Hidratos de carbono}

Las ingestas alimentarias de referencia de los hidratos de carbono se basan en la cantidad necesaria para suministrar glucosa a tejidos esenciales (como el cerebro) y no tienen en cuenta las necesidades de reemplazo del glucógeno muscular o hepático. En adultos, el rendimiento en el ejercicio de gran intensidad, prolongado o repetido, está vinculado a los depositos totales de glucógeno, dado que los hidratos de carbono son los combustibles limita- 
dores para mantener el ritmo de trabajo [30, 31]. En niños prepuberales se supone que la capacidad glucolítica es menos eficiente que en sujetos adolescentes o adultos, lo que induce a un incremento de la utilización de grasas durante el ejercicio prolongado en atletas jóvenes [32]. Todavía no se ha determinado si los competidores jóvenes se benefician o no de un nivel elevado de consumo de hidratos de carbono. La ingesta recomendada de hidratos de carbono es del 50 al $60 \%$ de la ingesta total diaria de energía, con sólo un 10\% de azúcares simples [33].

Los gimnastas y los bailarines de ballet creen que los hidratos de carbono son alimentos ricos en calorías. Aunque la ingesta media de hidratos de carbono en, por ejemplo, gimnastas, bailarines y patinadores artísticos, es del $\pm 50 \%$ de la ingesta total de energía, se basa predominantemente en azúcares simples en lugar de en hidratos de carbono complejos $[8,17,24,27,34]$. Estas encuestas nutricionales indican que la preferencia por los sabores dulces no se adapta bien al incremento de los depósitos de glucógeno musculares y hepáticos. En los deportes clasificados por pesos, las restricciones dietéticas podrían también inducir una reducción significativa del contenido en glucógeno muscular y hepático, precisamente antes de la competencia [5].

\section{Lípidos}

No se ha establecido ningún nivel de ingesta adecuada o ingesta diaria recomendada para los lípidos totales; sin embargo, existen niveles de ingesta adecuados con respecto a los ácidos grasos esenciales: ácidos linoléico y linolénico (n-3 y n-6; tabla 1 ).

Se recomienda que en niños y adolescentes el 30\% de la ingesta total de energía proceda de las grasas $[1,33]$. Aunque los niños utilizan proporcionalmente más grasa como fuente de energía durante el ejercicio prolongado que los adultos, no hay datos que permitan suponer que precisen un mayor consumo de lípidos.

En relación con la finalidad del control del peso o la restricción de peso, es habitual sugerir una reducción del consumo de lípidos. Una disminución de la ingesta total de energía a través de la reducción de las grasas puede constituir una estrategia peligrosa en atletas infantiles o adolescentes. De hecho, se exige una cantidad mínima de grasa corporal para garantizar las adaptaciones metabólicas y hormonales. El nivel recomendado para sustentar el estado de salud y la buena forma física es del 7\% en hombres y el 14\% en mujeres [35]. La reducción, o incluso la exclusión, de productos grasos es una aberración que implica deficiencias de grasas monoinsaturadas y poliinsaturadas. Además, productos grasos como los alimentos lácteos y la carne roja contienen proteína de gran ca-
Tabla 1. Niveles de ingesta adecuados de los ácidos linoléico y linolénico

\begin{tabular}{lll}
\hline \multirow{2}{*}{ Edad } & \multicolumn{2}{l}{ Ácido graso, g/día } \\
\cline { 2 - 3 } & ácido linoléico & ácido linolénico \\
\hline De 9 a 13 años & 12 & 1,2 \\
$\quad$ Niños & 10 & 1,0 \\
$\quad$ Niñas & & \\
De 14 a 18 años & 16 & 1,6 \\
$\quad$ Niños adolescentes & 11 & 1,1 \\
$\quad$ Niñas adolescentes & 11 \\
\hline
\end{tabular}

lidad, calcio, magnesio, hierro, cinc, cromo, vitamina $\mathrm{B}_{12}$, vitaminas liposolubles, etc., que son necesarios para un crecimiento óptimo. Todas las encuestas nutricionales sobre deportes estéticos, incluyendo bailarines de ballet, patinadores artísticos, gimnastas y gimnastas rítmicos, indican una ingesta de lípidos insuficiente $[8,11,17,24,27]$. En los deportes clasificados por pesos, la distribución de las grasas oscila ampliamente entre los períodos de 'pérdida de peso' e 'incremento de peso' (dieta 'yo-yo'). En consecuencia, el porcentaje de grasa corporal se modifica de forma perpetua, lo que probablemente trastorna el metabolismo de los lípidos durante la fase de crecimiento.

\section{Micronutrientes}

La ingesta insuficiente de energía se acompaña, en general, de ingestas marginales de micronutrientes (minerales y vitaminas). De hecho, en estudios en los que se trataba de que atletas jóvenes informasen sobre restricciones calóricas, los micronutrientes no fueron capaces de satisfacer las recomendaciones $[5,6,8,17,24,34,36$, 37]. En estudios dedicados a examinar la ingesta alimentaria en niños y adolescentes activos o en atletas jóvenes, se identifican corrientemente deficiencias de dos micronutrientes importantes, concretamente calcio y hierro. Estos micronutrientes son cruciales, no sólo para un buen estado de salud, sino también para optimizar el rendimiento atlético. El hierro actúa como componente de un cierto número de proteínas, entre las que destacan enzimas, hemoglobina y mioglobina. La importancia de esta última reside en el transporte de oxígeno a los tejidos de todo el organismo con fines metabólicos. La carencia de hierro induce anemia y deteriora el rendimiento. El calcio es también un micronutriente importante para el desarrollo de la masa ósea, un proceso capaz de proteger frente a la osteoporosis en la edad adulta. Más del 99\% del calcio corporal total se acumula en los dientes y los hue- 
sos. El resto está presente en la sangre, el líquido extracelular, el músculo y otros tejidos, donde desempeña un papel mediador de la contracción vascular y la vasodilatación, la contracción muscular, la transmisión neural y la secreción glandular.

La pubertad aumenta las necesidades de hierro y calcio debido a incrementos de la masa de hemoglobina, la deposición tisular, los brotes de crecimiento y el inicio de la menstruación en mujeres. En deportes con restricción de energía, el calcio y el hierro alimentarios son muy bajos $[38,39]$, dado que los atletas jóvenes rehuyen ingerir alimentos lácteos y carne roja por la preocupación que les suscita la ingesta de grasas o el deseo de seguir una dieta vegetariana.

\section{Líquidos y ejercicio}

El estado de hidratación previo a la participación en actividades físicas o deportes es un indicador importante del rendimiento y la resistencia, y puede limitar los traumatismos en adultos así como en niños o adolescentes. Se recomienda la ingesta de líquidos antes, durante o al final del ejercicio. Las deficiencias hídricas al final de la actividad física deben compensarse con arreglo a la pérdida de peso.

La información científica sobre los deportes clasificados por peso sigue siendo escasa con respecto a las estrategias de deshidratación. No obstante, desde un punto de vista práctico, estos atletas limitan la ingesta de todos los líquidos antes de la competencia (agua, sodas, café, sopa, etc.) y, además, dado que utilizan ropa interior sintética, generalmente sudan durante ejercicios intensivos para estimular la pérdida de agua y peso. Habitualmente se practican otras estrategias, como saunas, diuréticos y vómitos, con la intención de 'dar el peso' $[5,28]$. El beneficio esperado de las estrategias de restricción de líquidos y deshidratación aguda parece consistir en una rápida pérdida de peso que permite competir a los atletas en una categoría específica. Esta estrategia, tal como se demuestra a continuación, no carece de consecuencias.

\section{Consecuencias potenciales sobre la salud}

La dificultad al respecto reside en establecer la causalidad entre las restricciones de alimentos y líquidos y los efectos sobre la salud. De hecho, la interacción total entre factores genéticos y ambientales (como la nutrición) dificulta la demostración. No obstante, otros estudios respaldan la explicación de efectos potencialmente negativos sobre la salud, inducidos por la restricción de peso en atletas infantiles y/o adolescentes.

\section{Crecimiento y maduración}

A pesar de la talla corta 'normal' de los gimnastas de elite, los gimnastas rítmicos y los bailarines de ballet, y los evidentes beneficios de la actividad física sobre la salud durante el crecimiento, existen temores de que los competidores de 'deportes estéticos' que participan en programas de entrenamiento de gran nivel puedan conllevar el riesgo de efectos adversos sobre el crecimiento y la maduración. De hecho, en ausencia de una nutrición suficiente para compensar el mayor nivel de actividad física, las demandas energéticas del entrenamiento pueden competir por la energía disponible con las de los procesos celulares que subyacen al crecimiento y la maduración normales, especialmente durante de los picos de velocidad en el crecimiento del adolescente [40].

Por ejemplo, las gimnastas poseen una masa adiposa menor que los controles y son también significativamente más bajas y livianas para su edad, siendo más importantes las diferencias en gimnastas de más edad, de nivel avanzado [41]. Casi todo el tiempo, la maduración sexual se retrasa en gimnastas de ambos sexos, en comparación con sus gemelos y/o hermanos menos activos. Aunque los estudios de crecimiento longitudinales ofrecen más información que los datos transversales, tampoco demuestran la causalidad [42]. No obstante, en la mayoría de estos estudios se destaca un crecimiento atenuado y una maduración retrasada en atletas femeninas jóvenes que participan en programas de entrenamiento intensivos, y numerosos datos indican la puesta al día del crecimiento cuando la magnitud total del entrenamiento disminuye y/o cuando la alimentación es suficiente [ver los datos en 42]. Informes frecuentes indican restricciones de energía en atletas femeninas adolescentes de nivel competitivo avanzado. Este mecanismo podría explicar el efecto adverso sobre el crecimiento y la maduración, dado que el brote de crecimiento puberal es sensible a los factores nutricionales. Los mecanismos entre la restricción de alimentos y la atenuación del crecimiento y la maduración en niños y adolescentes se deben, en parte, a menores concentraciones circulantes de hormonas relacionadas con el crecimiento. De hecho, sabemos que las restricciones calóricas y proteínicas crónicas reducen el nivel del factor de crecimiento de tipo insulínico 1 (IGF-1) [43]. En comparación con controles o nadadores, los niveles plasmáticos de IGF-1 son menores en gimnastas femeninas jóvenes y se asocian a ingestas de energía insuficientes, maduración retrasada y crecimiento perturbado [44-46]. Por el contrario, las concentraciones plasmáticas de IGF-1 no se reducen en gimnastas masculinos prepuberales y en la fase inicial de la pubertad en 
comparación con los controles [47], si bien es bien sabido que la ingesta alimentaria limitada siempre es más marcada en las niñas.

\section{Composición corporal}

La composición corporal difiere en función del deporte. En deportes que imponen restricciones de peso, se observa variabilidad en las medidas relativas de grasa corporal y peso corporal magro entre deportes estéticos y deportes clasificados por peso, dado que en la segunda categoría aparecen fluctuaciones ponderales.

\section{Composición corporal en deportes estéticos}

Casi siempre, los atletas que participan en deportes en los que el aspecto físico es un factor importante para conseguir el éxito, son muy delgados, dado que inician conscientemente una dieta restringida para mantener un físico prepuberal, con peso corporal bajo y masa adiposa baja. En consecuencia, se dispone de una extensa literatura en la que se describe que los gimnastas, los gimnastas rítmicos y los bailarines de ballet prepuberales, al inicio de la pubertad y en edad escolar se caracterizan por un porcentaje bajo o muy bajo de grasa corporal [ver revisión en 48]. Paradójicamente, en estudios realizados en atletas o en personas ajenas a las actividades atléticas se ha demostrado que la respuesta adaptativa humana a la restricción de energía consiste en una reducción de índice metabólico en reposo, con un posible incremento de los depósitos adiposos [49]. Por este motivo, estos atletas se topan cada vez mas con mayores dificultades para mantener una masa adiposa corporal baja, y al final de la competición se suele observar una alteración en el tejido adiposo con incremento del tamaño total de las células adiposas y/o del número de dichas células [15].

En general, el desequilibrio energético generado por un nivel elevado de actividad física y restricción dietética se asocia a una menor densidad ósea [49]. No obstante, incluso si se reducen las ingestas de calcio en atletas estéticos, como gimnastas, patinadores artísticos, bailarinas de ballet o gimnastas rítmicos, estos jóvenes atletas de ambos sexos presentan una densidad del mineral óseo significativamente superior a la de otros grupos atléticos o grupos de control ajenos a actividades atléticas [48]. Tanto la genética como la tensión ejercida sobre el hueso (saltos y desmontes repetidos) contribuyen a elevar la densidad del mineral óseo. Por lo tanto, independientemente de las ingestas nutricionales, el mineral óseo, e incluso el tamaño óseo, aumenta en los atletas de elite que participan en actividades de alto impacto.

Restricción de peso en atletas jóvenes y consecuencias sobre la salud

\section{Composición corporal en deportes clasificados por pesos}

En deportes con categorías de pesos específicas, como la lucha, el judo, etc., las fluctuaciones ponderales son rápidas, frecuentes e importantes. Por lo tanto, la composición corporal puede modificarse con regularidad. Las pérdidas de peso a corto plazo se componen predominantemente de agua, grasa, proteína y glucógeno. Cuando los atletas pierden peso y almacenan grasa, la distribución de la grasa corporal puede cambiar. En el curso de la restricción energética, la contribución de las reservas de grasa a la pérdida total del peso aumenta considerablemente. No obstante, casi siempre, los atletas jóvenes se someten a una dieta rápida y pierden peso durante períodos cortos, lo que ocasiona pérdidas significativas de tejido magro y agua $[5,15]$. Reduciendo la magnitud de la deficiencia energética e incrementando la duración del período de pérdida de peso, se reduciría al mínimo la pérdida de tejido magro y se incrementaría al máximo la pérdida de grasa. Cuando los atletas pierden y recuperan peso, los depósitos de grasa pueden desaparecer y, a continuación, reaparecer en diferentes zonas corporales [15]. Si se pierde grasa en un lugar y se recupera en otro, la distribución de la grasa podría modificarse incluso si la grasa total fuera similar. Aunque poco se conoce de las pautas de incremento de peso en los primeros atletas clasificados por peso, posteriormente, la ingesta de la 'dieta yo-yo' podía inducir un cociente cintura/cadera elevado, indicativo del riesgo de enfermedades cardiovasculares, al igual que en personas obesas.

\section{Eficiencia nutricional}

La deficiencia nutricional puede definirse como el cociente entre el cambio de peso y las calorías ingeridas. Representa un índice de cuánto debe comer una persona para mantener un peso o una composición corporal dada. Si la eficiencia nutricional aumenta, disminuye la ingesta calórica necesaria para sustentar $1 \mathrm{~kg}$ de peso corporal. A partir de estudios diferentes, realizados para indagar las ingestas nutricionales y la composición corporal en atletas jóvenes de ambos sexos, Brownell et al. [15] plantearon la hipótesis de que la eficiencia nutricional podía incrementarse en atletas con peso bajo o fluctuante. Es probable que este mecanismo sea una adaptación fisiológica facilitadora de reservas de energía como proceso vital. No obstante, cuando aumenta la ingesta calórica, puede aparecer rápidamente un incremento de peso. En términos anecdóticos, algunas gimnastas se lamentan de que el mantener su peso bajo llega a ser cada

Ann Nestlé [Esp] 2006;64:77-84 
vez más difícil, hasta el punto de obligarlas a una lucha constante.

\section{Disfunción menstrual y capacidad reproductora}

Dixon et al. [50] dieron a entender que niveles altos de actividad física ocasionan un incremento de la secreción de endorfinas que, en combinación con una restricción calórica, causan una regulación anormal de la actividad hipotalámica. La función irregular del hipotálamo precipita la reducción de la frecuencia pulsátil de la secreción de gonadotropinas (hormona folículoestimulante y hormona luteinizante) e induce, a menudo, el retraso de la maduración puberal y, en consecuencia, oligomenorrea o amenorrea [8]. La regulación del hipotálamo y de la hipófisis varía considerablemente de un deporte a otro. Es bien sabido que las gimnastas de elite tienden a presentar un retraso en la edad de la menarquia, así como una irregularidad menstrual (amenorrea, oligomenorrea, anovulación, deficiencia de la fase luteínica) debido a alteraciones hormonales causadas por las dietas y el programa de entrenamiento [51]. Si bien la amenorrea representa la forma más extrema de disfunción menstrual, otras formas pueden dar lugar también a una supresión de los niveles de estrógenos y una influencia negativa sobre la salud ósea y la fertilidad. En consecuencia, las niñas físicamente activas y las adolescentes que realizan deportes estéticos pueden presentar en teoría la 'tríada de la atleta femenina', un síndrome constituido por tres componentes: trastornos del comer, amenorrea y osteoporosis (a pesar de que esta tercera sea menos habitual en actividades de alto impacto, tal como se ha demostrado anteriormente).

La supresión o la reducción de los niveles de testosterona en hombres puede ser el acontecimiento homólogo de la disfunción menstrual en mujeres. Strauss et al. [52] registraron niveles séricos de testosterona bajos en luchadores masculinos, sobre todo cuando el porcentaje de grasa corporal disminuía por debajo del 5\%. Los niveles regresaron a la normalidad después de la temporada, cuando se incrementaba el peso. Por lo tanto, el peso corporal bajo, la grasa corporal baja, la desnutrición y/o un nivel elevado de entrenamiento pueden representar una amenaza para la capacidad reproductora de los atletas masculinos.

\section{Estado del hierro y anemia}

En atletas entrenados se observan frecuentemente niveles bajos de hemoglobina en la sangre (13 a $14 \mathrm{~g} / 100 \mathrm{ml}$ en hombres y $12 \mathrm{~g} / 100 \mathrm{ml}$ en mujeres ), además de niveles bajos del hematocrito y la ferritina $(<20 \mu \mathrm{g} / \mathrm{l})$. Estos parámetros definen el concepto de 'anemia de causa deportiva' [53]. La masa de hemoglobina aumenta con la pubertad. Para esta finalidad y debido a la deposición tisular, en la época de crecimiento y el inicio de la menstruación en las niñas, las necesidades de hierro son más elevadas durante el período puberal [33]. Niveles bajos de hierro pueden deberse a hemolisis mecánica, hemorragia intestinal, hematuria, sudor, ingesta baja de hierro o absorción intestinal deficiente [53]. El menoscabo resultante del transporte de gases en la sangre y de la actividad de las enzimas musculares deteriora el rendimiento. Aunque el estado del hierro en atletas adolescentes se ha examinado sólo en pocos estudios, parece que estos atletas, en la vida adulta, son también propensos a una deficiencia de hierro no anémica $[54,55]$. Por otra parte, en gimnastas de ambos sexos se describen pruebas de vaciamiento de las reservas de hierro (niveles bajos de ferritina) [38].

\section{Traumatismos}

En numerosas investigaciones se ha revisado los traumatismos en jóvenes gimnastas de elite. En estudios retrospectivos y prospectivos se demuestra que las tasas de lesiones en gimnastas femeninas son elevadas y se asocian positivamente al nivel de rendimiento. Los traumatismos agudos (por ejemplo, fracturas por sobrecarga y esguinces ligamentosos) en los pies y los dedos de los pies son tan corrientes como los traumatismos en el tobillo [48]. Algunos de estos traumatismos agudos podrían deberse a la restricción alimentaria, dado que es bien sabido que los niveles bajos de estrógenos y las ingestas insuficientes de proteínas y calcio, en combinación con una menarquia tardía, ocasionan potencialmente un aumento de la tasa de fracturas por sobrecarga [56].

Durante las estrategias de pérdida de peso en deportes clasificados por peso, es habitual observar traumatismos musculoesqueléticos debidos a la privación de líquidos antes de la competición [35]. La posibilidad de sufrir una lesión térmica tampoco se descarta si la competición se realiza en un entorno intensamente cálido.

\section{Efectos de la deshidratación}

Una reducción intensa de peso podría deteriorar realmente las funciones fisiológicas. Por ejemplo, la deshidratación podría afectar a los riñones por reducción del flujo plasmático y la filtración. Entre los luchadores jóvenes se han descrito también casos de irritabilidad, estreñimien- 
to y 'sensación de abatimiento' [37]. Los efectos de una pérdida de peso rápida y la deshidratación sobre el rendimiento fisiológico están perfectamente confirmados [35]. Dichos efectos podrían consistir en: reducción de la fuerza muscular y la capacidad de potencia anaerobia; aumento de la frecuencia cardiaca en reposo y submáxima; reducción del volumen sistólico cardiaco, con subsiguiente disminución de la capacidad para mantener el trabajo a un ritmo constante; menor consumo de oxígeno; deterioro de los procesos de termorregulación; menores volúmenes plasmático y sanguíneo; depleción del glucógeno muscular y, posiblemente, del glucógeno hepático; problemas en la homeostasis de la glucosa; alteración del estado hormonal; reducción de la función inmunitaria, etc. [35]. Además, la investigación in vitro ha dejado entrever que la deshidratación de las células puede precipitar el inicio de una degradación de proteínas y glucógeno como medio para ajustar la osmolaridad celular en respuesta a una reducción del volumen del líquido intracelular [57].

\section{Trastornos del comer}

Los trastornos del comer pueden definirse como comer o no comer en respuesta a un impulso externo en lugar de un impulso interno. Los trastornos del comer, en la población atlética femenina, son merecedores de una atención creciente. Las atletas que participan en deportes que exigen delgadez y/o un peso corporal específico son más propensas a presentar trastornos del comer que otras atletas [58]. Por lo tanto, las gimnastas deportivas rítmicas competitivas, las gimnastas, las bailarinas de ballet y las patinadoras artísticas, por ejemplo, son acusadas de fomentar una reducción del peso poco fisiológica que puede progresar hasta manifestar anorexia y/o bulimia nerviosa [58]. Este hecho puede ser explicado por la enorme presión que reciben las atletas jóvenes para triunfar y conseguir un peso corporal ideal. En consecuencia, las atletas que practican deportes 'basados en la delgadez' describen una mayor insatisfacción corporal, una autoestima más baja y el seguimiento más persistente de dietas que otras atletas. La incapacidad para controlar el peso y la forma corporal puede dar lugar a frustración, culpabilidad e incluso desesperación, todo lo cual puede inducir a trastornos sociales y emocionales.

\section{Morbilidad y mortalidad}

Los procedimientos dietéticos seguidos por algunos atletas pueden incrementar el riesgo de padecer enferme- dades agudas o crónicas. Aparte de la característica específica de la dieta (grasas saturadas, azúcares simples, sodio, etc.), las pautas de peso corporal podrían influir también sobre el estado de salud. Es bien sabido que la restricción calórica crónica reduce el riesgo de ciertos cánceres [59] y atenúa la enfermedad coronaria, la hipertensión y la diabetes. No obstante, los atletas con bajo peso y trastornos del comer pueden presentar deficiencias nutricionales importantes (por ejemplo, vitaminas, minerales, aminoácidos esenciales, ácidos grasos esenciales) que pueden alterar las funciones metabólicas y orgánicas. Por ejemplo, las pacientes con anorexia nerviosa presentan una mayor dispersión de QT que las mujeres constitucionalmente delgadas y de peso normal, debido a niveles de potasio muy bajos (la dispersión de QT ha sido definida como la diferencia entre el intervalo QT más largo y el intervalo QT más corto que aparecen en el ECG de 12 derivaciones) [60]. Del mismo modo, las tandas de pérdidas y recuperaciones repetidas de peso pueden generar diferentes efectos negativos sobre el estado de salud. De hecho, la pérdida de peso se asocia al descenso de la presión arterial y el incremento de peso, a la elevación de aquella. Si el período de incremento es más importante que el período de disminución, la presión arterial puede afectarse. Además, en diferentes estudios se ha dado a entender que de las fluctuaciones del peso en luchadores puede derivar en una isquemia renal capaz de predisponerles a una hipertensión ulterior [15].

\section{Conclusión}

Tal como se demuestra en esta revisión, las consecuencias sobre el estado de salud de la restricción de peso impuesta por los deportes en la infancia son numerosas, y en ocasiones irreversibles. Para evitar estos efectos espectaculares, recomendamos monitorizar minuciosamente el estado nutricional de atletas jóvenes muy activos, independientemente de la edad, el deporte que practiquen y el sexo, especialmente los participantes en deportes que se caracterizan por una ingesta alimentaria limitada (deportes estéticos) o un consumo de alimentos irregular (deportes clasificados por pesos). Un enfoque psicológico y una educación nutricional para atletas, entrenadores, padres, etc. deberían completar el asesoramiento dietético a cargo de un especialista en nutrición. 


\section{Bibliografía}

1 DRI: Dietary Reference Intakes for Energy, Carbohydrate, Fiber, Fat, Fatty Acids, Cholesterol, Protein, and Amino Acids (Macronutrients). Washington, National Academy Press, 2002.

2 Tveit-Milligan P, Spindler AA, Nichols JE: Genes and gymnastics: a case study of triplets. Sports Med Training Rehab 1993;4:47-52.

$\checkmark 3$ Lindholm C, Hagenfeldt K, Ringertz BM: Pubertal development in elite juvenile gymnasts. Effects of physical training. Acta Obstet Gynecol Scand 1994; 73:269-273.

4 Steen SN, Brownell KD: Patterns of weight loss and regain in wrestlers: has the tradition changed? Med Sci Sports Exerc 1990;22:762-768.

5 Boisseau N, Vera-Perez S, Poortmans JR: Food and fluid intake in adolescent female judo athletes before competition. Pediatr Exerc Sci 2005; 17:62-71.

6 Oppliger RA, Steen SA, Scott JR: Weight loss practices of college wrestlers. Int J Sport Nutr Exerc Metab 2003;13:29-46.

7 Steen SN, Oppliger RA, Brownell KD: Metabolic effects of repeated weight loss and regain in adolescent wrestlers. JAMA 1988;260:47-50.

$\checkmark 8$ Weimann E, Witzel C, Schwidergall S, Bohles HJ: Peripubertal perturbations in elite gymnasts caused by sport specific training regimes and inadequate nutritional intake. Int J Sports Med 2000;21:210 215.

9 Theintz GE, Howald H, Allemann Y, Sizonenko PC: Growth and pubertal development of young female gymnasts and swimmers: a correlation with parental data. Int J Sports Med 1989;10:87-91.

10 Schwidergall S, Weimann E, Witzel C, Molenkamp $\mathrm{G}$, Brehl S, Bohles H: Nutritional behaviour of female and male high performance gymnasts. Wien Med Wochenschr 1988;148:243-244.

11 Lindholm C, Hagenfeldt K, Hagman U: A nutrition study in juvenile elite gymnasts. Acta Paediatr 1995;84:273-277.

12 Kiningham RB, Gorenflo DW: Weight loss methods of high school wrestlers. Med Sci Sports Exerc 2001;33:810-813.

13 Gurd B, Klentrou P: Physical and pubertal development in young male gymnasts. J Appl Physiol 2003 95:1011-1015.

14 Georgopoulos N, Markou K, Theodoropoulou A, Paraskevopoulou P, Varaki L: Growth and pubertal development in elite female rhythmic gymnasts. J Clin Endocrinol Metab 1999;84:4525-4530.

15 Brownell KD, Steen SN, Wilmore JH: Weight regulation practices in athletes: analysis of metabolic and health effects. Med Sci Sports Exerc 1987;19: 546-556.

16 Baxter-Jones AD, Helms P, Maffulli N, BainesPreece JC, Preece M: Growth and development of male gymnasts, swimmers, soccer and tennis players: a longitudinal study. Ann Hum Biol 1995;22: 381-394.

17 Boisseau N, Persaud C, Jackson AA, Poortmans JR: Training does not affect protein turnover in preand early pubertal female gymnasts. Eur J Appl Physiol 2005;94:262-267.

18 Fogelholm GM, Kukkonen-Harjula TK, Taipale SA, Sievänen HT, Oja P, Vuori IM: Resting metabolic rate and energy intake in female gymnasts, figure-skaters and soccer players. Int J Sports Med 1995; 16:551-556.

19 Bass S, Bradney M, Pearce G, Hendrich E, Inge K: Short stature and delayed puberty in gymnasts: influence of selection bias on leg length and the duration of training on trunk length. J Pediatr 2000;136 149-155.
20 Zonderland ML, Claessens AL, Lefevre J, Philippaerts R, Thomis M: Delayed growth and decreased energy intake in female gymnasts; in Armstrong $\mathrm{N}$ Kirby B, Welsman J (eds): Children and Exercise XIX: Promoting Health and Well-Being: Proceedings of the XIXth International Symposium of the European Group of Pediatric Work Physiology. London, E\&FN Spon, 1997, pp 553-556.

21 Poortmans JR: Protein metabolism; in Poortmans JR (ed): Principles of Exercise Biochemistry. Basel, Karger, 2004, pp 227-278

22 FAO/WHO: Joint Expert Committee: Energy and Protein Requirements. Geneva, World Health Organization, 1986.

23 Martin A: Apports nutritionnels conseillés pour la population française. Paris, Tec \& Doc, 2001

24 Cupisti A, D’Alessandro S, Castrogiovanni S, Barale E, Morelli E: Nutrition survey in elite rhythmic gymnasts. J Sports Med Phys Fit 2000;40:350 355.

25 Weimann E, Blum WF, Witzel C, Schwidergall S, Bohles HJ: Hypoleptinemia in female and male elite gymnasts. Eur J Clin Invest 1999;29:853-860.

26 Fogelholm GM, Kukkonen-Harjula TK, Taipale SA, Sievanen HT, Oja P, Vuori IM: Resting metabolic rate and energy intake in female gymnasts, figure-skaters and soccer players. Int J Sports Med 1995;16:551-556.

27 Benson J, Gillien DM, Bourdet K, Loosli AR: Inadequate nutrition and chronic calorie restriction in adolescent ballerinas. Phys Sportmed 1985;13:79-90.

28 Horswill CA, Park SH, Roemmich JN: Changes in the protein nutritional status of adolescent wrestlers. Med Sci Sports Exerc 1990;22:599-604.

29 Friedlander AL, Braun B, Pollack M, MacDonald JR, Fulco CS: Three weeks of caloric restriction alters protein metabolism in normal-weight, young men. Am J Physiol Endocrinol Metab 2005;289: E446-E455.

30 Coyle EF, Coyle E. Rating carbohydrates that speed recovery from training. Phys Sportsmed 1993;21 $111-123$

31 Costill DL, Sherman WM, Fink WJ, Maresh C, Witten M, Miller JM: The role of dietary carbohydrate in muscle glycogen resynthesis after strenuous running. Am J Clin Nutr 1981;34:1831-1836.

32 Boisseau N, Delamarche P: Metabolic and hormonal responses to exercise in children and adolescents. Sports Med 2000;30:405-422.

33 ANC: Apports nutritionnels recommandés (ANC) pour la population française. Paris, Tec \& Doc 2001.

34 Delistraty DA, Reisman EJ, Snipes MG: A physiological and nutritional profile of young female figure skaters. J Sports Med Phys Fit 1992;32:149-155.

35 Oppliger RA, Case HS, Horswill CA, Landry GL, Shelter AC: American College of Sports Medicine position stand. Weight loss in wrestlers. Med Sci Sports Exerc 1996;28:ix-xii.

36 Moffatt RJ: Dietary status of elite female high school gymnasts: inadequacy of vitamin and min eral intake. J Am Diet Assoc 1984;84:1361-1363.

37 Freischlag J: Weight loss, body composition, and health of high school wrestlers. Phys Sportmed 1984; $12: 121-126$

38 Constantini NW, Eliakim A, Zigel L, Yaaron M, Falk B: Iron status of highly active adolescents: evidence of depleted iron stores in gymnasts. Int J Sport Nutr Exerc Metab 2000;10:62-70.

39 Kirchner EM, Lewis RD, O’Connor PJ: Bone min eral density and dietary intake of female college gymnasts. Med Sci Sports Exerc 1995;27:543-549.
40 Borer KT: The effects of exercise on growth. Sports Med 1995;20:375-397.

41 Caine D, Lewis R, O’Connor P, Howe W, Bass S Does gymnastics training inhibit growth of females? Clin J Sport Med 2001;11:260-270.

42 Caine D, Bass S, Daly R: Does elite competition in hibit growth and delay maturation in some gymnasts? Quite possibly. Pediatr Exerc Sci 2003;15 360-372.

43 Smith WJ, Underwood LE, Clemmons DR: Effects of caloric or protein restriction on insulin-like growth factor-I (IGF-I) and IGF-binding proteins in children and adults. J Clin Endocrinol Metab 1995;80:443-449.

44 Theintz GE: Endocrine adaptation to intensive physical training during growth. Clin Endocrinol 1194;41:267-272.

45 Jahreis G, Kauf E, Frohner G, Schmidt HE: Influence of intensive exercise on insulin-like growth factor I, thyroid and steroid hormones in female gymnasts. Growth Regul 1991;1:95-99.

46 Bass S, Pearce G, Bradney M, Hendrich E, Delmas PD: Exercise before puberty may confer residual benefits in bone density in adulthood: studies in active prepubertal and retired female gymnasts. J Bone Miner Res 1998;13:500-507.

47 Daly RM, Rich PA, Klein R, Bass SL: Short stature in competitive prepubertal and early pubertal male gymnasts: the result of selection bias or intense training? J Pediatr 2000;137:510-516

48 O'Connor PJ, Lewis RD, Boyd A: Health concerns of artistic women gymnasts. Sports Med 1996;21: 321-325

49 Deutz RC, Benardot D, Martin DE, Cody MM: Relationship between energy deficits and body composition in elite female gymnasts and runners. Med Sci Sports Exerc 2000;32:659-668.

50 Dixon G, Eurman P, Stern BE, Schwartz B, Rebar RW: Hypothalamic function in amenorrheic runners. Fertil Steril 1984;42:377-383.

51 Manore MM: Dietary recommendations and athletic menstrual dysfunction. Sports Med 2002;32: 887-901

52 Strauss RH, Lanese RR, Malarkey WB: Weight loss in amateur wrestlers and its effect on serum testosterone levels. JAMA 1985;254:3337-3338.

53 Chatard JC, Mujika I, Guy C, Lacour JR: Anaemia and iron deficiency in athletes. Practical recom mendations for treatment. Sports Med 1999;27: 229-240.

54 Rowland TW: Iron deficiency in the young athlete. Pediatr Clin North Am 1990;37:1153-1163.

55 Rowland TW, Stagg L, Kelleher JF: Iron deficiency in adolescent girls. Are athletes at increased risk? J Adolesc Health 1991;12:22-25.

56 Benson JE, Geiger CJ, Eiserman PA, Wardlaw GM: Relationship between nutrient intake, body mass index, menstrual function, and ballet injury. J Am Diet Assoc 1989;89:58-63.

57 Petrie HJ, Stover EA, Horswill CA: Nutritional concerns for the child and adolescent competitor. $\mathrm{Nu}$ trition 2004;20:620-631.

58 Sundgot-Borgen J: Eating disorders, energy intake, training volume, and menstrual function in highlevel modern rhythmic gymnasts. Int J Sport Nutr 1996;6:100-109.

59 Kritchevsky D, Weber MM, Buck CL, Klurfeld DM: Calories, fat and cancer. Lipids 1986;21:272-274.

60 Franzoni F, Mataloni E, Femia R, Galetta F: Effect of oral potassium supplementation on QT dispersion in anorexia nervosa. Acta Paediatr 2002;91: 653-656. 